\title{
DIVERSITY AND DISTRIBUTION OF MICROFUNGI FROM DIPTEROCARP FORESTS IN SARAWAK, BORNEO ISLAND (MALAYSIA).
}

Keanekaragaman dan Distribusi mikrofungi dari hutan dipterokarpa di Sarawak, Pulau Borneo (Malaysia).

\author{
*Lateef A. A. ${ }^{1,2}$, Sepiah, M. ${ }^{1}$, and Bolhassan, M. H. ${ }^{1}$ \\ 1 Department of Plant Science and Environmental Ecology, Faculty of Resource Science and Technology, Universiti \\ Malaysia Sarawak, 94300 Kota Samarahan, Sarawak, Malaysia. \\ ${ }^{2}$ Department of Plant Biology, Faculty of Life Science, University of Ilorin, Nigeria. \\ * Corresponding author: Lateef A.A. lateef.aa@ unilorin.edu.ng, +(60)146902928. \\ Received: 22 Mac $2016 \quad$ Revise: 22 Aug $2016 \quad$ Accepted:19 Sept 2016
}

\begin{abstract}
South-east Asian dipterocarp forests contain plant species that are vulnerable and at risk of extinction. Microfungi, as important decomposers and sources of useful metabolites, have not been well studied in regards to their diversity and distribution in the South-east Asian dipterocarp forests. This study reports the microfungal species associated with dominant and vulnerable plant species in two dipterocarp forests, namely Gunung Gading and Kubah National Parks, in Sarawak on the Borneo Island. Green leaves and litters of five host plants, namely Baccaurea sp., Macaranga triloba (Thunb.) Müll.Arg., Macaranga sp., Shorea macrophylla (de Vr.) Ashton and Syzygium sp., were incubated on water agar and malt extract agar. A total of 171 fungal taxa were recorded on the five host plants, during two visits, each to both forests. Syzygium sp. had the highest microfungal diversity of 84 taxa followed by 80 taxa on Macaranga sp., 53 taxa on Baccaurea sp., 43 taxa on M. triloba and the lowest, 35 taxa, on Shorea macrophylla. Pestalotiopsis spp., Oidiodendron spp., Graphium spp., and Mycelia sterilia were the most frequently isolated microfungal species from all the five host plants. There was a high microfungal similarity between Syzygium sp. and Macaranga sp. with 34 taxa common to both plants. The lowest similarity was recorded between S. macrophylla, M. triloba and Baccaurea sp. with only 13 taxa common between them. This is the first report on microfungi associated with dominant and vulnerable plant species in South-east Asian dipterocarp forests; an important record of the fungal diversity on these plants and an exposition on microfungi in Sarawak, Borneo Island, Malaysia.
\end{abstract}

Keywords: Microfungi, Macaranga, Shorea, Baccaurea, Syzygium

\begin{abstract}
ABSTRAK Selatan-timur hutan dipterokarpa Asia berisi spesies tanaman yang rentan dan terancam punah. Mikrofungi, sebagai dekomposer penting dalam ekosistem dan sumber metabolit yang berguna belum diteliti dengan baik dalam hal keragaman dan distribusinya di hutan dipterokarpa Asia Tenggara. Penelitian ini mengungkapkan spesies mikrofungi terkait dengan spesies tanaman yang dominan dan rentan dalam dua hutan dipterokarpa, yaitu Gunung Gading dan Taman Nasional Kubah, di Sarawak di pulau Borneo. Daun hijau dan Serasah dari lima tanaman inang diinkubasi pada agar air dan ekstrak malt agar. Sebanyak 171 taksa mikrofungi dicatat pada lima tanaman inang, yaitu Baccaurea sp., Macaranga triloba, Macaranga sp., Shorea macrophylla dan Syzygium sp. selama empat kunjungan ke kedua hutan. Syzygium sp. telah keragaman mikrofungal tertinggi dari 84 taksa diikuti oleh 80 taksa pada Macaranga sp., 53 taksa pada Baccaurea sp., 43 taksa di M. triloba dan terendah, 35 taksa, pada Shorea macrophylla. Pestalotiopsis spp., Oidiodendron spp., Graphium spp., dan miselia steril yang spesies mikrofungal yang paling sering diisolasi dari semua lima tanaman inang. Ada kesamaan microfungal tinggi antara Syzygium sp. dan Macaranga sp. dengan 34 taksa umum untuk kedua tanaman. Kesamaan terendah tercatat antara S. macrophylla, M. triloba dan Baccaurea sp. dengan hanya dengan 13 taksa umum untuk ketiganya. Ini adalah laporan pertama pada mikrofungi terkait dengan spesies tanaman rentan dan dominan di hutan dipterokarpa Asia Tenggara; catatan penting dari keragaman mikrofungi didukung oleh tanaman ini dan dalam pameran mikrofungi di Sarawak, pulau Borneo, Malaysia.
\end{abstract}

Kata kunci: Mikrofungi, Macaranga, Shorea, Baccaurea, Syzygium

\section{INTRODUCTION}

The forest ecosystem is home to a vast diversity of plants, animals and microorganisms (Engelbrecht, 2012) including fungi. Forests are essential to the long-term wellbeing of local populations, national economies, and the earth's biosphere as a whole (Santiago Declaration, 1995). Dipterocarp forests, mainly found in the South-east Asian region (Whitmore, 1975), are 
unique and have been noted for their high diversity of living organisms. These dipterocarp forests got their name from the plant family (Dipterocarpaceae) which dominates the forests' plant species. Among the major plant families found in this type of forest are the Dipterocarpaceae, Phyllanthaceae, Myrtaceae and Euphorbiaceae. In Malaysia, the dipterocarp forest is one of the major forest types in the country, occupying about $80 \%$ of the total natural forests in the Malaysian state of Sarawak on the Borneo Island (Sarawak Forestry, 2015).

Microfungi are known for their various importance to human life, for example, as natural decomposers, source of novel drugs, biological control of pests and diseases, in industrial production of chemicals and also as part of our ecosystem diversity. Microfungi on different plant species of the dipterocarp forests on the Borneo Island have not been well documented. Also, there is insufficient report on the microfungal communities associated with many plant families, including the Dipterocarpaceae, Phyllanthaceae, Myrtaceae and Euphorbiaceae which are found in the dipterocarp forests in the tropics and in other forest types across the globe. Few studies on microfungi associated with plants in dipterocarp forests include that of Osono et al., (2009) on Shorea obtusa, Bettucci \& Roquebert (1995) on S. robusta and recently Harahap, Rahayu, \& Hidayat (2013) on litters of Shorea spp.

Understanding and monitoring the diversity and distribution of fungi in our forests and on various plant families are necessary for the proper functioning of our ecosystem in addition to being sources of sustainable and renewable raw materials for human uses. This study was carried out in order to understand the diversity and distribution of microfungi associated with five host plants in two dipterocarp forests in Sarawak, Borneo Island, Malaysia. The result from this study will be a valuable contribution to the global fungal diversity in reference to their distribution on plants and their occurrence in dipterocarp forests and in Sarawak, Borneo Island.

\section{MATERIALS AND METHODS}

\section{The Sample Collection Sites}

The sample collection sites were Kubah National Park and Gunung Gading National Park located in the seventh division of Sarawak, East Malaysia. The two Parks are covered with a lowland mixed dipterocarp forest, with the family Dipterocarpaceae dominating the plant species and are characterised by a tropical climate with an average annual temperature of $27{ }^{\circ} \mathrm{C}$ and an average annual rainfall of 4095 mm (Climate-Data, 2015).

\section{Sampling Design}

Leaf samples were collected from five host plants namely Syzygium sp., Baccaurea sp., Shorea macrophylla, Macaranga triloba, and Macaranga sp. on four collection trips to both sampling sites; two trips to Kubah National Park in March, 2014 and September, 2014, likewise, two trips to Gunung Gading National Park in November, 2014 and February, 2015. The host plants studied were selected based on their availability at the site since the vegetation at each site is natural, and not planted, thus the plants grow at random points. The latitudinal and longitudinal readings at each collection point were recorded with a handheld Global Positioning System (GPS) equipment (Table 1). 
Table 1. Details of the host plants sampled in this study

\begin{tabular}{|c|c|c|c|c|}
\hline & Plant collected & Collection site & GPS co-ordinate & Elevation \\
\hline 1. & Macaranga triloba (Family Euphorbiaceae) & KNP & $\begin{array}{l}\mathrm{N} 01^{\circ} 36081 \\
\text { E } 110^{\circ} 11204\end{array}$ & 410 \\
\hline 2. & Shorea macrophylla (Family Dipterocarpaceae). & KNP & $\begin{array}{l}\text { N } 01^{\circ} 36757 \\
\text { E } 110^{\circ} 11750\end{array}$ & $175 \mathrm{~m}$ \\
\hline 3. & Baccaurea sp. (Phyllanthaceae) & GNP & $\begin{array}{l}\mathrm{N} 01^{\circ} 41510 \\
\text { E } 110^{\circ} 50704\end{array}$ & $635 \mathrm{~m}$ \\
\hline 4. & Macaranga sp. (Family Euphorbiaceae) & GNP & $\begin{array}{l}\mathrm{N} 01^{\circ} 41510 \\
\text { E } 109^{\circ} 50704\end{array}$ & $635 \mathrm{~m}$ \\
\hline 5 . & Syzygium sp. (Family Myrtaceae) & GNP & $\begin{array}{l}\text { N } 01^{\circ} 41625 \\
\text { E } 109^{\circ} 50573\end{array}$ & $657 \mathrm{~m}$ \\
\hline
\end{tabular}

KNP=Kubah National Park

GNP= Gunung Gading National Park

For each plant sampled, leaves were cut from different parts of the plant, mostly matured leaves while leaf-litters were picked directly around the plant on the ground. The samples were put in plastic bags and transported to the laboratory for processing.

\section{Processing of Leaf Samples and Data analysis}

Upon return to the laboratory, the leaves were processed within $24 \mathrm{hrs}$ for endophytic and

saprophytic microfungal isolation as described in Lateef, Sepiah, \& Bolhassan (2015). Endophytic fungi were isolated from leaves which were washed under running tap water to remove superficial dust and debris on their surface, cut into $1 \mathrm{~cm}^{2}$ and then surface sterilized with $70 \%$ ethanol for one minute, 10 $\%$ hydrogen peroxide $\left(\mathrm{H}_{2} \mathrm{O}_{2}\right)$ for five minutes, rinsed with $70 \%$ ethanol for one minute and finally rinsed with deionized sterile distilled water. For saprophytic microfungi isolation, the leaf samples were washed with double sterilized distilled water, cut into $1 \mathrm{~cm}^{2}$ and washed again with sterile distilled water. The leaf segments were allowed to dry fully under sterile conditions in laminar flow before incubating on the media. Four hundred leaf segments were used from each plant species except for S. macrophylla in which only 200 leaf segments were incubated without any antibiotics on water agar and malt extract agar. Identification of the microfungi were made to genus level, and wherever possible, to species level based on conidia types and colony appearance using various identification guides (Carmichael, Kendrick, Connors, \& Sigler, 1980; Ellis, 1971, 1976; Kendrick \& Carmichael, 1973; Morris, 1963; Seifert, Morgan-Jones, Gams, \& Kendrick, 2011).

Data on the occurrences of the microfungi on the leaf segments were recorded and the presence of a microfungus on each leaf segment was recorded as one occurrence. The frequency of isolation of each microfungal taxa was determined according to Hata \& Futai (1995) and Osono (2008) as follows:

Frequency of isolation $($ I. F. $)=$ the total number of leaf segments from which a fungal taxa was present

the total number of leaf segments observed 
Diversity index such as the Shannon's diversity index as well as species accumulation curves and species richness were estimated with two richness estimators - Abundance-base Coverage Estimator (ACE) and Chaol using the EstimateS diversity software (http://purl.oclc.org/estimates) version 9.1.0 released June 2013 (Colwell, 2013).

\section{RESULTS AND DISCUSSION}

\section{Microfungal Diversity}

One hundred and seventy-one microfungal taxa were recovered, which included 29 ascomycetes, seven basidiomycetes, 21 coelomycetes, 102 hyphomycetes, five zygomycetes and seven sterile forms from a total of 1800 leaf fragments of the five host plants namely $M$. triloba, Macaranga sp. S. macrophylla, Baccaurea sp. and Syzygium sp. (Table 2). The highest number of microfungal species, eighty-four (84) taxa, was recorded on the host plant Syzygium sp. followed by 80 taxa on Macaranga sp., 53 taxa on Baccaurea sp., 43 on $M$. triloba and the lowest, 35 taxa, on Shorea macrophylla (Table 3).

Table 2. Percentage (\%) occurrence and host plant distribution of fungal taxa on leaves of plants from Kubah and Gunung Gading National Parks in Sarawak (“_“" means absent).

\begin{tabular}{|c|c|c|c|c|c|c|}
\hline & & ost plant & & & & \\
\hline & Fungal species & $\begin{array}{l}\text { Syzygium } \\
\text { sp. }\end{array}$ & $\begin{array}{l}\text { Baccaurea } \\
\text { sp. }\end{array}$ & $\begin{array}{l}\text { Shorea } \\
\text { macrophylla }\end{array}$ & $\begin{array}{l}\text { Macaranga } \\
\text { triloba }\end{array}$ & $\begin{array}{l}\text { Macaranga } \\
\text { sp. }\end{array}$ \\
\hline 1 & Acremonium spp. & - & 0.25 & 2 & 8.5 & 7 \\
\hline 2 & Anthostomella sp. & 1.5 & - & - & - & - \\
\hline 3 & Arthrinum sp. & 0.25 & - & - & - & - \\
\hline 4 & Ascomycete 1 & - & - & - & - & 0.5 \\
\hline 5 & Ascomycete 17 & - & - & 2.5 & - & - \\
\hline 6 & Ascomycete 2 & - & - & - & - & 0.25 \\
\hline 7 & Ascomycete 26 & - & - & 0.5 & - & - \\
\hline 8 & Ascomycete 3 & - & - & 0.5 & - & - \\
\hline 9 & Ascomycete 4 & - & 0.25 & - & - & - \\
\hline 10 & Aspergillus niger & 0.25 & 1.5 & - & 0.25 & 0.5 \\
\hline 11 & Aspergillus sp. & 0.5 & - & - & 1.25 & - \\
\hline 12 & Aureobasidium pullulans & - & - & - & 0.25 & 1 \\
\hline 13 & Basidiomycete 2 & - & - & - & - & 0.25 \\
\hline 14 & Basidiomycete 3 & 5 & 0.25 & - & - & - \\
\hline 15 & Basipetospora sp. & 0.75 & - & - & - & - \\
\hline 16 & Beltrania rhombica & 0.25 & 3.75 & - & 0.5 & 5.25 \\
\hline 17 & Beltraniopsis sp. & - & - & - & - & 1.5 \\
\hline 18 & Bipolaris sp. & - & - & - & - & 0.75 \\
\hline 19 & Bispora sp. & - & 0.25 & - & 1 & - \\
\hline 20 & Black Mycelia sterilia 1 & 4.5 & 7.75 & 14 & 12.5 & 13.25 \\
\hline 21 & Black Mycelia sterilia 2 & - & - & - & 1.75 & 0.75 \\
\hline
\end{tabular}




\begin{tabular}{|c|c|c|c|c|c|c|}
\hline 22 & Blakeslea sp. & - & - & - & - & 0.75 \\
\hline 23 & Botryodiplodia sp. & 0.25 & - & 0.5 & 0.75 & 5.5 \\
\hline 24 & Botryodiplodia theobromae & - & - & 2 & - & 6.75 \\
\hline 25 & Botrytis sp. & - & - & 2.5 & - & - \\
\hline 26 & Brachyphoris sp. & - & - & - & - & 0.5 \\
\hline 27 & Brown Mycelia sterilia & 0.5 & 1 & - & - & 0.75 \\
\hline 28 & Campylospora sp. & 0.25 & 0.25 & - & - & - \\
\hline 29 & Cercospora sp. & 1.25 & - & - & 1.25 & - \\
\hline 30 & $\begin{array}{l}\text { Ceriospora } \\
\text { polygonacearum }\end{array}$ & 1.25 & - & - & - & - \\
\hline 31 & Chaetomium sp. & - & - & 1.5 & - & 1.25 \\
\hline 32 & Chaetopsina sp. & - & - & - & 0.25 & - \\
\hline 33 & Chaetospermum artocarpi & 0.75 & 1.75 & - & - & - \\
\hline 34 & Chaetospermum spp. & 0.25 & 0.25 & - & - & 2 \\
\hline 35 & Chaetosphaeria sp. & 11.75 & - & - & - & 1 \\
\hline 36 & Chloridium sp. & - & - & - & - & 2.75 \\
\hline 37 & Circinotrichum fertile & 0.25 & - & - & 0.5 & - \\
\hline 38 & Circinotrichum sp. 2 & 2.5 & - & - & - & - \\
\hline 39 & Circinotrichum sp. 3 & 1.5 & - & - & - & - \\
\hline 40 & Cladosporium sp. & 4 & 0.75 & - & - & 6 \\
\hline 41 & Codinae sp. & 1.75 & 3.25 & - & 0.5 & - \\
\hline 42 & Coelomycete 1 & - & - & - & 0.25 & - \\
\hline 43 & Coelomycete 2 & - & - & - & 2.5 & - \\
\hline 44 & Coelomycete 3 & - & 0.5 & - & - & - \\
\hline 45 & Coelomycete 39 & - & 0.75 & - & - & - \\
\hline 46 & Coelomycete 54 & 1.25 & - & - & - & - \\
\hline 47 & Colletotrichum acutatum & 1.25 & - & 4.5 & 4.5 & - \\
\hline 48 & $\begin{array}{l}\text { Colletotrichum } \\
\text { gloeosporioides }\end{array}$ & 12.75 & 5.5 & 9 & 8.25 & - \\
\hline 49 & Colletotrichum sp. & 0.5 & - & - & - & - \\
\hline 50 & Conidiobolus-like sp. & - & - & - & - & 1.25 \\
\hline 51 & Cordana sp. & 0.5 & - & - & - & - \\
\hline 52 & Cryptophiale kakombensis & - & - & - & 0.5 & - \\
\hline 53 & Cryptophiale minor & - & - & - & 1 & - \\
\hline 54 & Cryptophiale sp. & - & - & - & 0.25 & - \\
\hline 55 & Cryptophiale udagawae & 0.25 & - & - & 0.75 & - \\
\hline 56 & Curvularia sp. & - & - & - & - & 0.75 \\
\hline 57 & $\begin{array}{l}\text { Cylindrocarpon } \\
\text { obtusisporum }\end{array}$ & - & - & - & 0.5 & - \\
\hline 58 & Cylindrocarpon sp. & 0.5 & - & - & - & - \\
\hline
\end{tabular}




\begin{tabular}{|c|c|c|c|c|c|c|}
\hline 59 & Cylindrocladium sp. 3 & 4.25 & - & 0.5 & - & 1.25 \\
\hline 60 & Dactylaria ciliata & 2.5 & - & - & 0.75 & 1.75 \\
\hline 61 & Dactylaria obtriangularia & 1.75 & 2 & - & - & - \\
\hline 62 & Dactylaria sp. 1 & - & - & - & - & 8.75 \\
\hline 63 & Dactylaria sp. 2 & - & 0.25 & - & - & 4 \\
\hline 64 & Dictyosporium sp. 1 & - & - & - & - & 3 \\
\hline 65 & Dictyosporium sp. 2 & - & 0.75 & - & - & - \\
\hline 66 & Didymella sp. 3 & - & - & - & - & 4.5 \\
\hline 67 & Diplococcium sp. & - & - & - & 1 & - \\
\hline 68 & Diplodia sp. & 1.75 & 1.25 & - & - & 1.25 \\
\hline 69 & Fusariella sp. & - & 0.5 & - & - & 1.25 \\
\hline 70 & Fusarium spp. & 1 & - & 0.5 & 6 & 3.75 \\
\hline 71 & Gelasinospora sp. & 1.25 & - & - & - & - \\
\hline 72 & Gliocladium sp. & 2 & - & - & - & - \\
\hline 73 & $\begin{array}{l}\text { Glomerella sp. (Sexual } \\
\text { state of Colletotrichum) }\end{array}$ & 0.75 & - & - & - & 1.25 \\
\hline 74 & Gnomonia sp. & 6.75 & 3.25 & 11 & 7 & - \\
\hline 75 & Gonatobotrys sp. & 0.5 & - & - & - & - \\
\hline 76 & Grahium pennicillioides & 16 & 22.25 & 8 & 2.5 & 6.5 \\
\hline 77 & Graphium sp. 2 & 5.5 & - & - & - & - \\
\hline 78 & Guemannomyces graminis & - & 0.5 & - & - & 9.25 \\
\hline 79 & Hansfordia sp. & 4.5 & - & - & 1.5 & 2.5 \\
\hline 80 & Helicomyces sp. & 1.5 & 2.5 & - & - & 4.5 \\
\hline 81 & Helicosporium sp. & 0.5 & - & - & - & 1 \\
\hline 82 & Humicola grisea & 0.25 & 8.75 & 0.5 & - & - \\
\hline 83 & Humicola sp. & 0.75 & - & - & - & 0.5 \\
\hline 84 & Hyphomycete 1 & - & - & - & 2 & - \\
\hline 85 & Hyphomycete 3 & - & - & 1.5 & - & - \\
\hline 86 & Hyphomycete 4 & 0.5 & - & - & - & - \\
\hline 87 & Hyphomycete 49 & - & - & - & - & 0.75 \\
\hline 88 & Hyphomycete 54 & - & - & - & - & 3 \\
\hline 89 & Hyphomycete 7 & 0.25 & - & - & - & - \\
\hline 90 & Idriella lunata & - & - & - & - & 0.25 \\
\hline 91 & Isthmolongispora minima & 1 & - & - & - & - \\
\hline 92 & Isthmotricladia laeensis & 1.25 & - & - & - & - \\
\hline 93 & Kinochaeta sp. & 1.25 & - & - & 1.5 & 1.25 \\
\hline 94 & Kinochaeta-like sp. & 0.75 & - & - & - & - \\
\hline 95 & Kylindria sp. & 0.25 & - & - & - & 1.5 \\
\hline 96 & Lateriramulosa uniinflata & - & - & - & - & 0.25 \\
\hline
\end{tabular}




\begin{tabular}{|c|c|c|c|c|c|c|}
\hline 97 & $\begin{array}{l}\text { Leptospahaeria } \\
\text { horniaraensis }\end{array}$ & - & 0.5 & 4 & - & 3.25 \\
\hline 98 & Leptosphaeria sp. 2 & - & 0.5 & - & - & - \\
\hline 99 & Lophiostoma sp. & - & - & - & - & 1 \\
\hline 100 & Lophiostoma sp. 2 & - & - & - & - & 1.5 \\
\hline 101 & Menispora sp. & 0.5 & - & - & - & - \\
\hline 102 & Minimedusa sp. & 2.25 & 0.5 & - & - & 3.5 \\
\hline 103 & Monacrosporium sp. & 1.5 & - & - & - & 6.75 \\
\hline 104 & Monilinia sp. & - & - & 2.5 & - & 0.75 \\
\hline 105 & Monodictys sp. & - & - & - & - & 0.25 \\
\hline 106 & Mucor sp. & 1 & - & 9.5 & 12.5 & - \\
\hline 107 & Mucor sp. 2 & - & - & - & 2.75 & - \\
\hline 108 & Myxomycete 1 & - & - & - & 1 & - \\
\hline 109 & Neopestalotiopsis sp. & - & - & - & - & 2.25 \\
\hline 110 & Neottiosporella sp. & - & 2 & - & - & - \\
\hline 111 & Oidiodendron sp. & 11.75 & 12.5 & 2.5 & 12.5 & 4.75 \\
\hline 112 & Ojibwaya-like sp. & - & - & - & - & 0.5 \\
\hline 113 & Paecilomyces sp. & 0.75 & - & - & - & 1 \\
\hline 114 & Penicillium sp. & 1.75 & 10.25 & 1 & - & 0.5 \\
\hline 115 & Penicillium sp. 2 & 1.5 & - & - & - & - \\
\hline 116 & Periconia sp. & 0.25 & - & - & - & - \\
\hline 117 & Pestalotiopsis humus & - & - & 5 & - & - \\
\hline 118 & Pestalotiopsis malayana & 3 & - & - & - & - \\
\hline 119 & Pestalotiopsis sp. & 5.5 & 45.25 & 13.5 & - & 15 \\
\hline 120 & Pestalotiopsis sp. 15 & - & - & 3.5 & - & - \\
\hline 121 & Pestalotiopsis sp. 16 & - & - & 0.5 & - & - \\
\hline 122 & Pestalotiopsis sp. 17 & - & - & 1 & - & - \\
\hline 123 & Pestalotiopsis sp. 7 & - & 0.25 & - & - & - \\
\hline 124 & Pestalotiopsis sp. 9 & - & - & 10.5 & - & - \\
\hline 125 & Phalangispora sp. & 3 & - & - & - & - \\
\hline 126 & Phialophora sp. & - & 0.25 & - & - & - \\
\hline 127 & Phomopsis sp. & 0.75 & 4.25 & - & - & 0.75 \\
\hline 128 & Physarum sp. & - & - & - & - & 1 \\
\hline 129 & Pink Mycelia sterilia & - & 0.25 & - & - & 1.75 \\
\hline 130 & $\begin{array}{l}\text { Pseudopestalotiopsis } \\
\text { kubahensis }\end{array}$ & - & - & - & 3.25 & - \\
\hline 131 & Ramularia sp. & - & 0.25 & - & 0.25 & - \\
\hline 132 & Rhinocladiella cristaspora & 3.25 & 3.5 & - & - & 5 \\
\hline 133 & Rhinocladiella sp. & - & - & - & 1.75 & - \\
\hline 134 & Rhinocladium sp. & 1.5 & 0.25 & - & - & - \\
\hline
\end{tabular}




\begin{tabular}{|c|c|c|c|c|c|c|}
\hline 135 & Sartorya sp. & - & 0.75 & - & - & - \\
\hline 136 & Scolecobasidium sp. & 2.25 & - & 1.5 & - & 15 \\
\hline 137 & Septonema sp. & - & - & - & - & 1.25 \\
\hline 138 & Speiropsis pedatospora & 2.25 & - & - & - & 0.25 \\
\hline 139 & Sporidesmium sp. & - & - & - & 1 & - \\
\hline 140 & Sporidesmium sp. 2 & - & - & - & - & 0.25 \\
\hline 141 & Sporochisma nigroseptata & - & 0.25 & - & - & - \\
\hline 142 & Stachybotrys sp. & 0.75 & - & - & - & - \\
\hline 143 & Stachybotrys sp. 1 & - & - & 2.5 & 2.5 & - \\
\hline 144 & Stachybotrys sp. 2 & - & 0.5 & - & - & - \\
\hline 145 & Stibella sp. & - & - & - & - & 6.75 \\
\hline 146 & Suttoniella sp. & - & - & - & - & 1.25 \\
\hline 147 & Sympodiella sp. & - & 2.25 & - & - & 0.5 \\
\hline 148 & Thielavia sp. & 0.5 & - & $1-$ & - & - \\
\hline 149 & Thozetella sp. & 0.25 & - & 1 & - & - \\
\hline 150 & Torula sp. & - & 0.25 & - & - & 3.5 \\
\hline 151 & Torula sp. 2 & - & - & - & - & 1.25 \\
\hline 152 & Trichoderma koningii & - & - & - & - & - \\
\hline 153 & Trichoderma sp. & 8.75 & 5.25 & 22 & 0.25 & - \\
\hline 154 & Trichoderma viride & 0.5 & - & - & - & - \\
\hline 155 & Triscelophorus sp. & 0.25 & - & - & - & 1.5 \\
\hline 156 & Ulocladium sp. & - & - & - & - & 3.5 \\
\hline 157 & Ulocladium sp. 2 & - & 0.5 & - & - & - \\
\hline 158 & Unidentified 3 & - & - & - & - & 2.5 \\
\hline 159 & Venturia sp. & - & - & - & 3.25 & - \\
\hline 160 & Verticillium sp. & 7 & 0.75 & - & 0.5 & 21.25 \\
\hline 161 & Verticillium sp. 3 & 0.5 & - & - & - & - \\
\hline 162 & Volutella ciliata & 1 & - & - & - & - \\
\hline 163 & Volutella sp. & 0.25 & - & - & - & - \\
\hline 164 & White Mycelia sterilia 1 & 7.75 & 10.5 & 7 & 0.25 & 11.25 \\
\hline 165 & White Mycelia sterilia 3 & - & 0.75 & - & - & - \\
\hline 166 & White Mycelia sterilia 7 & - & - & - & - & 0.25 \\
\hline 167 & Wiesneriomyces javanicus & 3 & 2.25 & - & - & 0.25 \\
\hline 168 & Wiesneriomyces-like sp. & - & - & - & - & 5.75 \\
\hline 169 & Xylaria sp. & 0.5 & 0.5 & - & - & - \\
\hline 170 & Yellow Mycelia sterilia & 0.25 & 1.75 & 0.5 & - & - \\
\hline 171 & Zygosporium sp. & - & - & - & - & 0.25 \\
\hline
\end{tabular}


Table 3. Diversity indices and species estimate of microfungi based on host plant.

\begin{tabular}{llllccccc}
\hline $\begin{array}{c}\text { Name of Plant } \\
\text { host }\end{array}$ & $\begin{array}{l}\text { No. of } \\
\text { isolates }\end{array}$ & $\begin{array}{l}\text { Species } \\
\text { observed }\end{array}$ & Singletons Doubletons & $\begin{array}{c}\text { ACE Chao1(SE*) } \\
\text { (SE*) }\end{array}$ & $\begin{array}{c}\text { \% } \\
\text { SC* }\end{array}$ & $\begin{array}{c}\text { Shannon } \\
\text { Index }\end{array}$ \\
\hline Syzygium sp. & 749 & 84 & 17 & 13 & 93.7 & 93.7 & 89.65 & 3.79 \\
Baccaurea sp. & 707 & 53 & 14 & 9 & 62.09 & 62.09 & 85.35 & 2.91 \\
S. macrophylla & 319 & 35 & 8 & 3 & 41.98 & 41.98 & 83.37 & 3.04 \\
M. triloba & 447 & 43 & 8 & 6 & 46.99 & 46.99 & 91.50 & 3.12 \\
Macaranga sp. & 993 & 80 & 10 & 7 & 85.62 & 85.62 & 93.43 & 3.81 \\
\hline
\end{tabular}

SC*=Sample completeness

$\mathrm{SE}^{*}=$ Species estimate.

This study is the first detailed investigation of microfungi associated with several plant species in a dipterocarp forests in Borneo Island, Malaysia. The use of two different growth media in this study enhanced the isolation of different microfungal taxa. Using different media for microfungal diversity studies as earlier reported by Bills \& Polishook (1991) and Paulus, Kanowski, Gadek, \& Hyde (2006) helps to isolate both the slow growing and fast growing microfungi. In the present study, more microfungal taxa were isolated from Syzygium sp. from Gunung Gading National Park as compared to the other plant species. This is in line with previous reports that plant species in the family Myrtaceae, which Syzygium sp. belongs support high microfungal diversity (Cheewangkoon et al., 2009) which can be attributed to their possession of certain oils and other substances which favour occurrence of diverse microfungal communities (Carnegie, Burgess, Beilharz, \& Wingfield, 2007; Crous, Mohammed, Glen, Verkley, \& Groenewald, 2007).

Macaranga sp. and $M$. triloba are dominant plant species in disturbed forests in Southeast Asia (Whitmore, 1975) and in this study, they were more abundant in Kubah National Park compared to Gunung Gading National Park. Eighty and forty-three microfungal taxa were recorded from Macaranga sp. from Kubah and M. triloba from Gunung Gading respectively. The difference in the microfungal diversity observed from these two plant species of the same genus can be attributed to the different environmental factors in the two sites, and this phenomenon has been earlier reported by Paulus (2004) that site environmental characteristics have influence on microfungal diversity on closely related plant species.

With regards to Baccaurea sp. and $S$. macrophylla, inventory of microfungal species on the leaves of these plants has not been reported. Shorea macrophylla is presently a vulnerable species at risk of extinction and is mainly found in the South-east Asian forests as a source of quality timber. Documenting the microfungi on this vulnerable plant species at risk of biological extinction is important with many implications. Similar studies on fungal diversity on close relatives of $S$. macrophylla were carried out on $S$. obtusa, S. robusta and Shorea spp. by Osono et al., (2009), Bettucci \& Roquebert (1995) and recently by Harahap et al., (2013), respectively in which low water content and presence of certain phenolic compounds were suggested to be responsible for the low fungal colonization on Shorea spp.

The Chao 1 estimates of species richness on each plant species using the diversity software EstimateS ranged from 93.7 taxa on leaves of Syzygium sp. to 41.98 taxa on leaves of S. macrophylla (Table 3). The Chao 1 species estimate for all the plant hosts studied indicated that some microfungal species were 
missed during the isolation period; though this is usual in most microfungal surveys as the Chao 1 and ACE species richness prediction are always higher than the observed species richness (Mohamed \& Martiny, 2011; Muthukrishnan, 2012; Zhao et al., 2015).

Species accumulation curves (SACs) of each plant species did not reach an asymptote (Figure 1), suggesting that there are more species to be discovered from these plants. However, the sampling completeness ranged from $83.37 \%$ for S. macrophylla to as high as $93.43 \%$ for Macaranga sp. (Table 3) showing that the samples collected were enough to recover majority of the microfungal species present on the leaves of the studied plant species, though the sampling was not totally exhaustive.

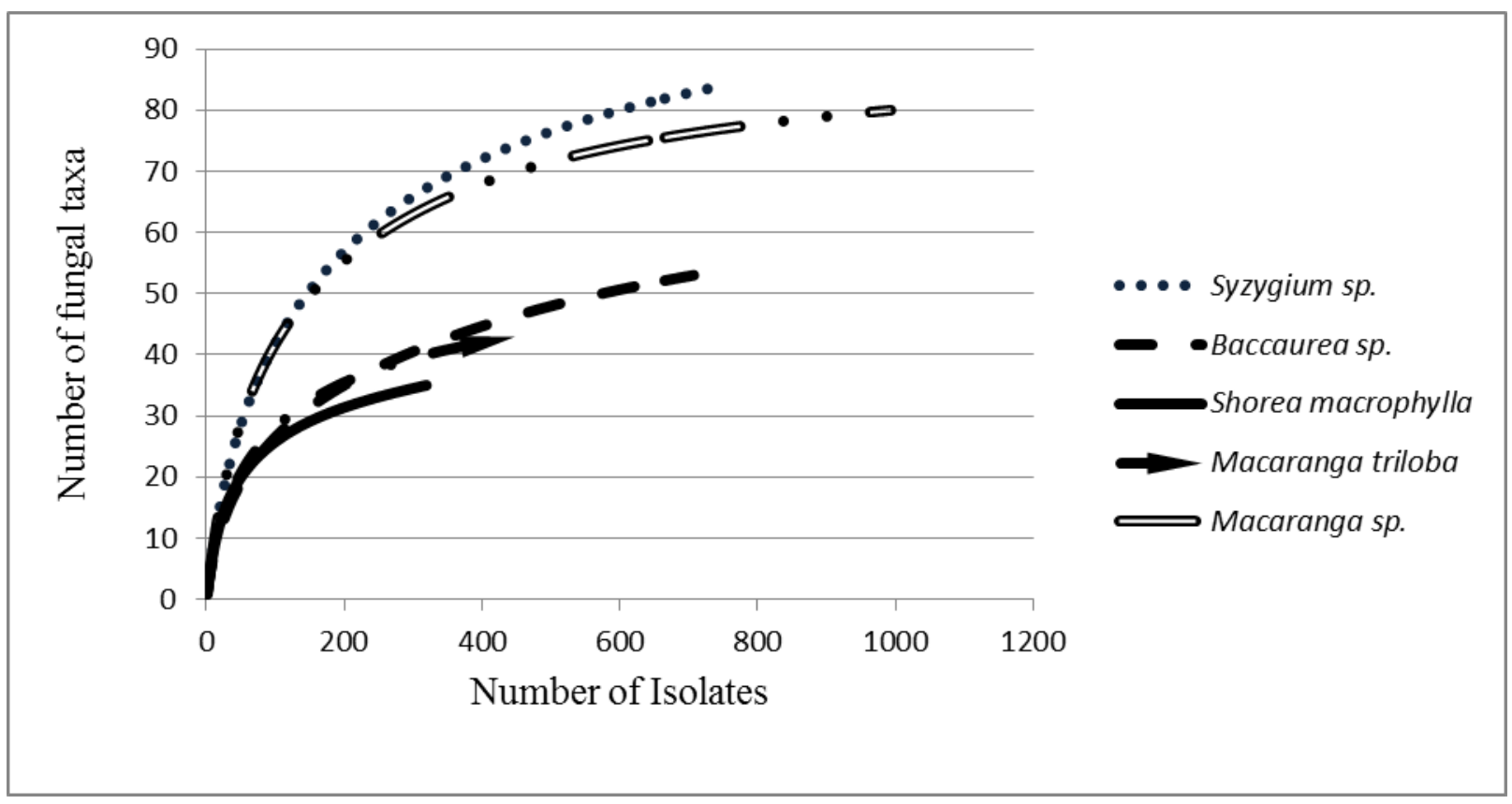

Figure 1. Rarefied species accumulation curves (SACs) of the five host plant studied

\section{Microfungal Distribution and similarities between the host plants}

Out of the 171 fungal taxa recorded, only four taxa were isolated from all the five host plants, six taxa from four host plants while majority of the microfungi were recorded only on one host plant. Majority of the microfungal taxa $(67 \%)$ that were recorded only on one host plant were isolated from Syzygium sp. Four fungal taxa (Black Mycelia sterilia, Oidiodendron sp., white Mycelia sterilia and Graphium penicillioides) were common on all the five plant species studied. Mycelia sterilia (both white and black forms) are known to be generalist fungi found on a wide range of host plants (Allegrucci, Bucsinszky, Arturi, \& Cabello, 2015; Kumar, 2013; Sepiah, Roha, \& Laman, 2006) while Oidiodendron sp. and Graphium penicillioides have also been isolated from a variety of host plants. This result shows that these microfungal species can be recovered from most plant species.

The most frequently isolated taxa from the host plants belonged to the genera Pestalotiopsis, Oidiodendron, Graphium, Mycelia sterilia, Trichoderma, Colletotrichum, 
Verticillium, Gnomonia, Mucor, Scolecobasidium, Acremonium, Penicillium, Chaetosphaeria, Rhinocladiella, Fusarium and Cladosporium (Figure 2). Pestalotiopsis spp. were isolated from $85 \%$ of all the five host plants' leaves while Colletotrichum spp. were recorded on 55\% of all the five host plants' leaves. Pestalotiopsis spp. are known common plant saprophytes and endophytes (Maharachchikumbura, Hyde, Groenewald, Xu, $\&$ Crous, 2014). Together with $C$. gloeosporioides and Trichoderma spp., Pestalotiopsis spp. have been reported as one of the most frequently occurring microfungi on different host plant (Cannon \& Simmons, 2002; Sepiah et al., 2006). Other species have also been reported as frequent species such as Penicillium sp. on 15 medicinal plants in India and bamboo leaf respectively (Kumar, 2013; Shankar, Shashikala, \& Krishnamurthy, 2008), Acremonium sp., Verticillium sp., Fusarium sp. (Kumar, 2013; Shankar et al., 2008). However, Gnomonia sp., Scolecobasidium sp. and Rhinocladiella sp. were also isolated frequently in this study, which were not common in previous studies.

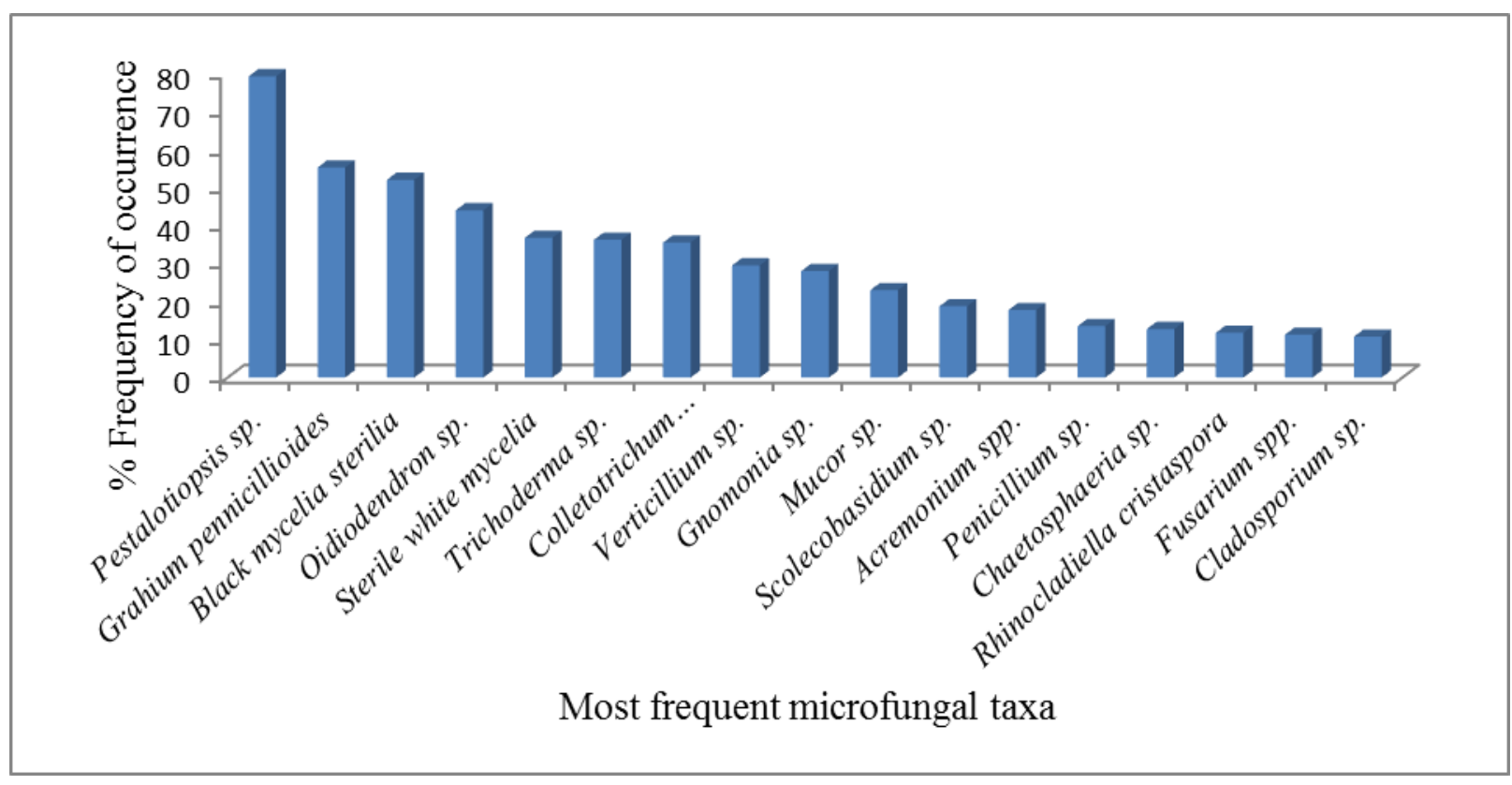

Figure 2. The most frequently observed fungal taxa from all the five host plants studied.

Table 4. Shared microfungal species/overlap between different host plants.

Host plant

Syzygium sp.

Macaranga sp.

Baccaurea sp.

M. triloba

S. macrophylla

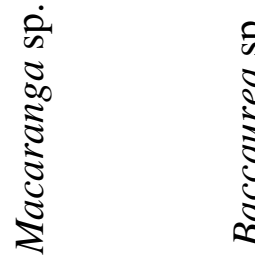

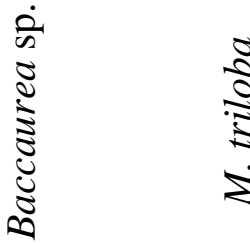

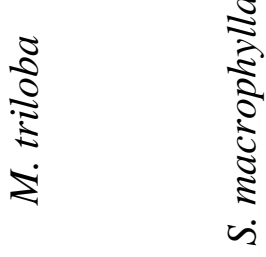

0

34

30

26

15

15

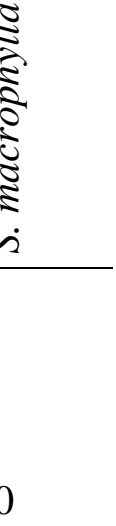


In terms of similarity of microfungal communities on the host plants, the highest microfungal similarities were between Syzygium sp. and Macaranga sp. in which they shared 34 taxa in common (Table 4).

However, there were low similarities between the microfungal communities on $S$. macrophylla, Macaranga sp. and Baccaurea sp. with only 13 taxa in common. The high microfungal community similarity between Syzygium sp. and Macaranga sp., both from Gunung Gading National Park signifies that they both share some similar fungal species. This could be a site-dependent occurrence, as the same high similarities were observed between all the host plants in Gunung Gading National Park. On the other hand, there was a low overlap in the microfungal communities on host plants from Kubah National Park suggesting that each host plant species in that site has its own adapted environment for its microfungal species. Furthermore, this also suggests that Gunung Gading's environment is still more natural than that of Kubah National Park which has been highly open to many human interactions as evidenced by the high human activities observed during our sample collection in Kubah National Park.

\section{CONCLUSION}

The microfungal communities found on Syzygium sp., M. triloba, Macaranga sp., Baccaurea sp., and S. macrophylla represent valuable resources about the mycoflora associated with these plants as well as Kubah and Gunung Gading National Parks in Borneo Island, Sarawak. The present fungal discoveries represent new records of microfungi from dominant plant species as well as vulnerable plant species from the South-east Asian dipterocarp forests. The results obtained in this study from each plant species can be compared with other host plants in the same plant family and with other plant species thereby increasing our understanding of the diversity and distribution of microfungi in the tropics.

\section{ACKNOWLEDGEMENTS}

The first author is grateful to Universiti Malaysia Sarawak and the Sarawak government for the Zamalah scholarship awarded to him and permission to collect samples from the National Parks respectively.

\section{REFERENCES}

Allegrucci, N., Bucsinszky, A. M., Arturi, M., \& Cabello, M. N. (2015). Communities of anamorphic fungi on green leaves and leaf litter of native forests of Scutia buxifolia and Celtis tala: Composition, diversity, seasonality and substrate specificity. Revista Iberoamericana de Micología, 32(2), 71-78. http://doi.org/10.1016/j.riam.2013.11.002

Bettucci, L., \& Roquebert, M.-F. (1995). Microfungi from tropical rainforest litter \& soil. Nova Hedwigia, 61(1-2), 111-118.

Bills, G. F., \& Polishook, J. D. (1991). Microfungi from Carpinus caroliniana. Canadian Journal of Botany, 69(7), 14771482. Retrieved from http://www.nrcresearchpress.com/doi/abs/1 0.1139/b91-191

Cannon, P. F., \& Simmons, C. M. (2002). Diversity and host preference of leaf endophytic fungi in the Iwokrama Forest Reserve, Guyana. Mycologia, 94(2), 210220. Retrieved from http://www.mycologia.org/content/94/2/210 .short

Carmichael, J. W., Kendrick, W. B., Connors, I. L., \& Sigler, L. (1980). Genera of Hyphomycetes. Edmonton, Canada: The University of Alberta Press.

Carnegie, A. J., Burgess, T. I., Beilharz, V., \& Wingfield, M. J. (2007). New species of Mycosphaerella from Myrtaceae in 
plantations and native forests in eastern Australia. Mycologia, 99(3), 461-474.

Cheewangkoon, R., Groenewald, J. Z., Summerell, B. A., Hyde, K. D., To-anun, C., \& Crous, P. W. (2009). Myrtaceae, a cache of fungal biodiversity. Persoonia, 23, $55-85$.

http://doi.org/10.3767/003158509X474752

Colwell, R. K. (2013). EstimateS: Statistical estimation of species richness and shared species from samples. Version 9 and earlier. User's Guide and application. http://purl.oclc.org/estimates. Retrieved from http://purl.oclc.org/estimates

Crous, P. W., Mohammed, C., Glen, M., Verkley, G. J. M., \& Groenewald, J. Z. (2007). Eucalyptus microfungi known from culture. 3. Eucasphaeria and Sympoventuria genera nova, and new species of Furcaspora, Harknessia, Heteroconium and Phacidiella. Fungal Diversity, 25(1), 19$36 . \quad$ Retrieved from http://www.fungalbiodiversitycentre.com/i mages/ResearchGroups/Phytopathology/pdf 125-2.pdf

Ellis, M. B. (1971). Dematiaceous Hyphomycetes. Kew, UK.: The Commonwealth Mycological Institute.

Ellis, M. B. (1976). More Dematiaceous Hyphomycetes. Kew, UK.: The Commonwealth Mycological Institute.

Engelbrecht, B. M. (2012). Plant ecology: Forests on the brink. Nature, 491(7426), 675-677.

Harahap, I., Rahayu, G., \& Hidayat, I. (2013). Community Structure of Sporulating Fungi on Decaying Litters of Shorea spp. Microbiology Indonesia, 7(3), 105-112.

Hata, K., \& Futai, K. (1995). Endophytic fungi associated with healthy pine needles and needles infested by the pine needle gall midge, Thecodiplosis japonensis. Canadian Journal of Botany. http://doi.org/10.1139/b95-040

Kendrick, W. B., \& Carmichael, J. W. (1973). Hyphomycetes. In G. C. Ainsworth, F. K. Sparrow, \& A. S. Sussman (Eds.), The fungi, A taxonomic review with keys:
Ascomycetes and fungi imperfecti (Vol. IVA, pp. 323-509). New York: Academic Press.

Kumar, R. (2013). Fungal diversity associated with bamboo litter from Bambusetum of Rain Forest Research Institute, Northeast India. Biodiversitas, Journal of Biological Diversity, $\quad$ 14(2), 79-88. http://doi.org/10.13057/biodiv/d140205

Lateef, A., Sepiah, M., \& Bolhassan, M. H. (2015). Microfungal diversity on leaves of Eusideroxylon zwageri, a threatened plant species in Sarawak, Northern Borneo. Biodiversitas, Journal of Biological Diversity, 16(2), 264-268. http://doi.org/10.13057/biodiv/d160222

Maharachchikumbura, S. S. N., Hyde, K. D., Groenewald, J. Z., Xu, J., \& Crous, P. W. (2014). Pestalotiopsis revisited. Studies in Mycology, 79, 121-186. http://doi.org/10.1016/j.simyco.2014.09.005

Mohamed, D. J., \& Martiny, J. B. H. (2011). Patterns of fungal diversity and composition along a salinity gradient. The ISME Journal, $5(3)$, 379-388. http://doi.org/10.1038/ismej.2010.137

Morris, E. F. (1963). The synnematous genera of the fungi imperfecti. USA: Western Illinois University.

Muthukrishnan, S. (2012). Species composition, seasonal changes and community ordination of alkalotolerant micro fungal diversity in a natural scrub jungle ecosystem of Tamil Nadu, India. Mycosphere, 3(2), 92-109. http://doi.org/10.5943/mycosphere/3/2/1

Osono, T. (2008). Endophytic and epiphytic phyllosphere fungi of Camellia japonica: seasonal and leaf age-dependent variations. Mycologia, 100(3), 387-391. http://doi.org/10.3852/07-110R1

Osono, T., Ishii, Y., Takeda, H., Seramethakun, T., Khamyong, S., To-Anun, C., ... Kakishima, M. (2009). Fungal succession and lignin decomposition on Shorea obtusa leaves in a tropical seasonal forest in northern Thailand. Fungal Diversity, 36(10). Retrieved from 
http://www.fungaldiversity.org/fdp/sfdp/FD 36-7.pdf

Paulus, B. C. (2004). The diversity and distribution of microfungi on leaf litter of an Australian wet tropics rainforest. James Cook University, Australia., Australia.

Paulus, B. C., Kanowski, J., Gadek, P. A., \& Hyde, K. D. (2006). Diversity and distribution of saprobic microfungi in leaf litter of an Australian tropical rainforest. Mycological Research, 110(12), 14411454.

http://doi.org/10.1016/j.mycres.2006.09.002

Santiago Declaration. (1995). Statement on criteria and indicators for the conservation and sustainable management of temperate and boreal forests. Journal of Forestry, 93(4): 18-21.(4), 18-21.

Sarawak Forestry. (2015). Sustainable Forest Management. Retrieved July 26, 2015, from http://www.sarawakforestry.com/

Seifert, K. A., Morgan-Jones, G., Gams, W., \& Kendrick, W. B. (2011). The genera of hyphomycetes. Netherlands: CBS-KNAW Fungal Biodiversity Center.

Sepiah, M., Roha, L., \& Laman, C. (2006). The Microfungi. In The Biodiversity of a Peat Swamp Forest in Sarawak. Edited by Fatimah Abang and Idraneil Das (p. 142). Kuching, Malaysia: Unit Penerbitan, Universiti Malaysia Sarawak.

Shankar, N. B., Shashikala, J., \& Krishnamurthy, Y. L. (2008). Diversity of fungal endophytes in shrubby medicinal plants of Malnad region, Western Ghats, Southern India. Fungal Ecology, 1(2-3), 89-93.

http://doi.org/10.1016/j.funeco.2008.05.001

Whitmore, T. C. (1975). Tropical rain forests of the far east. Oxford: Clarendon Press.

Zhao, M., Zhang, D., Su, X., Duan, S., Wan, J., Yuan, W., ... Pan, Y. (2015). An integrated metagenomics/metaproteomics investigation of the microbial communities and enzymes in solid-state fermentation of pu-erh tea. Scientific Reports, 5. http://doi.org/10.1038/srep10117 